\title{
The Design of High Performance Asynchronous Pipelines with Quasi Delay Insensitive
}

\author{
D. Jayanthi \\ Associate Professor, Dept. of ECE \\ Sona College of Technology, Salem.
}

\author{
M. Rajaram \\ Vice-Chancellor \\ Anna University of Technology, Tirunelveli
}

\begin{abstract}
VLSI Design looking towards to solve design constructions that arises when using clock pulses. The majority of the constrains can be overcome by using asynchronous logic, additionally synchronous circuits has some inherent advantages over synchronous counterparts. This paper demonstrates the design of efficient asynchronous pipelines for some standard logic circuits and medium scale integration (MSI) circuits. The performance analysis of various templates is designed for different standard logics and MSI circuits. The QDI templates are highly tolerant of process variations due to the up and down transitions are sensed. QDI circuits are quite robust in terms of process variations and design tolerances. In this work, expose a timing assumption used in staticizers for QDI logic and apply it to other parts of circuits. Goal of this work is to optimize circuits with respect to area and power while maintaining the robustness.
\end{abstract}

\section{Keywords}

Asynchronous Channels, QDI Templates PCHB, PCFB and RSPCHB.

\section{INTRODUCTION}

Asynchronous design which replaces [1] [2]clocking local handshaking has the potential to make high speed design more feasible Hence facilitating modular and reusable design .So that the localized control

of asynchronous pipeline is an excellent match for very high throughput fine grain data path. Asynchronous design which replaces [3] global clocking local handshaking has the potential to make high speed design more feasible. Asynchronous design has recently attracted industry [4] [5] due to its potential for low power consumption, low electromagnetic interfaces, high speed and robust interfacing. The focus of this paper is on a key enabling technology to make high speed asynchronous system practical, and the design of high throughput asynchronous pipelines. In the design of asynchronous systems, the global clock is eliminated and replaced [6] with local handshake channels which only communicate when and where necessary. Among the protocols used for handshaking, the micropipeline concept is first proposed in. An Asynchronous pipeline style introduced [7] for high speed application, the pipeline uses standard blocks of standard logic for processing data and single level sensitive SR latches to separate data items. This approach includes[8] a novel high-concurrent handshake protocol, with fewer synchronization points between neighboring pipeline stages than almost all existing asynchronous dynamic pipelining approaches. This study presents a methodology for improving the speed of high-speed adders. As a starting point, a previously proposed method, called "speculative completion," is used in which fastterminating additions are automatically detected. Unlike the previous design, the method proposed in this study is able to adapt dynamically to (1) Application-Specific Behavior and (2) The latches worked on AMULET1 are level sensitive, so two- to four-phase converters are necessary in each latch controller. To avoid this transparency, an investigation has been carried out into four-phase micropipeline control circuits; this has terrified up several design issues telling to cost, performance and safety and forms a useful illustration of asynchronous design techniques. A robust asynchronous full adder design [9] corresponding to early output logic, synthesized using the elements of a standard cell library is presented in this study. As the name suggests, the adder ensures the gate orphan freedom and neatly fits into the self-timed system architecture. In comparison with many of the indicating full adder designs, which can be embedded in the self-timed system, it is found. These design estimates correspond to simulation results of the 32-bit carry-ripple adder circuit; derived by targeting a high speed $130 \mathrm{~nm}$ bulk CMOS process technology. Also, the proposed full adder facilitates a faster reset and the return-to-zero for the fundamental carry-propagate topology is achieved with only two full adder delays. A QDI system is constructed as a collection of concurrent hardware modules (called processes) [10] that communicate with each other through message-passing channels. These messages consist of atomic data items called tokens, which are usually multirail encoded each process can send and receive tokens to and from other processes through one-to-one communication by means of handshake protocols. Due to the lack of global clock and multi-rail encoded data communication, QDI circuits have the potential to achieve self-checking and halt the circuit in the presence of failures. An asynchronous width adaptive data architecture that [11] activates operations only in significant data portions and adjusts the position of sign bit dynamically. This architectural approach [12] yields good average-case performance and reduces power consumption, but the representative implementation is based on the quasi-delayinsensitive asynchronous design style, which is known to be area expensive.

\section{Asynchronous channels}

An asynchronous communication channel is a bundle of wires and a protocol to communicate data between a sender and a receiver. The encoding scheme in which one wire per bit is used to transmit the data and an associated request line is sent to identify when data is valid is called single-rail encoding and is shown in 


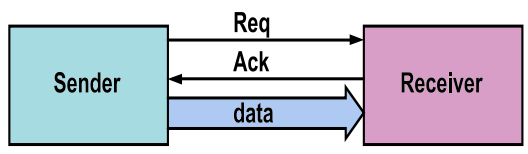

A) Abstract channel

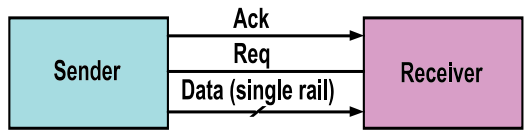

B) Bundled data channel

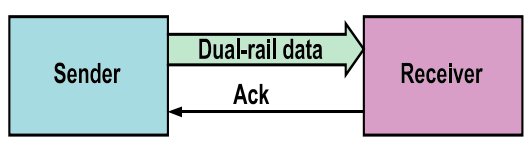

C) Dual rail channe

Fig 1: Hand shakes protocol

Fig. 1.A protocol that can be sending by sender through channel, this is called push channel, the opposite the receiver asking for new data is called pull channel. In both cases the directions of the request and acknowledge signals are reversed and the validity of data is indicated in the acknowledge signals from the sender to the receiver. The associated channel is called a bundled-data channel. Alternatively, if the data is sent using two wires for each bit of information, the encoding is called a dual rail channel. Extensions to 1-of-N encoding also exist. Both single-rail and dual-rail encoding schemes are commonly used and there are tradeoffs between each. Dual-rail and 1of-N encodings allow for data validity to be indicated by the data itself and are often used in QDI designs. Singlerail, in contrast, requires the associated request line, driven by a matched delay line, to always be longer than the computation. This latter approach requires careful timing analysis but allows the reuse of synchronous single-rail logic.

\section{MATERIALS AND METHODS}

PCHB: The Fig.2 below shows the template for PreCharged Half Buffer (PCHB).The test for validity and neutrality is confirmed using an input end detector Weakconditioned half buffer(WCHB).This input end detector is denoted by LCD while its output end detector is denoted by RCD.

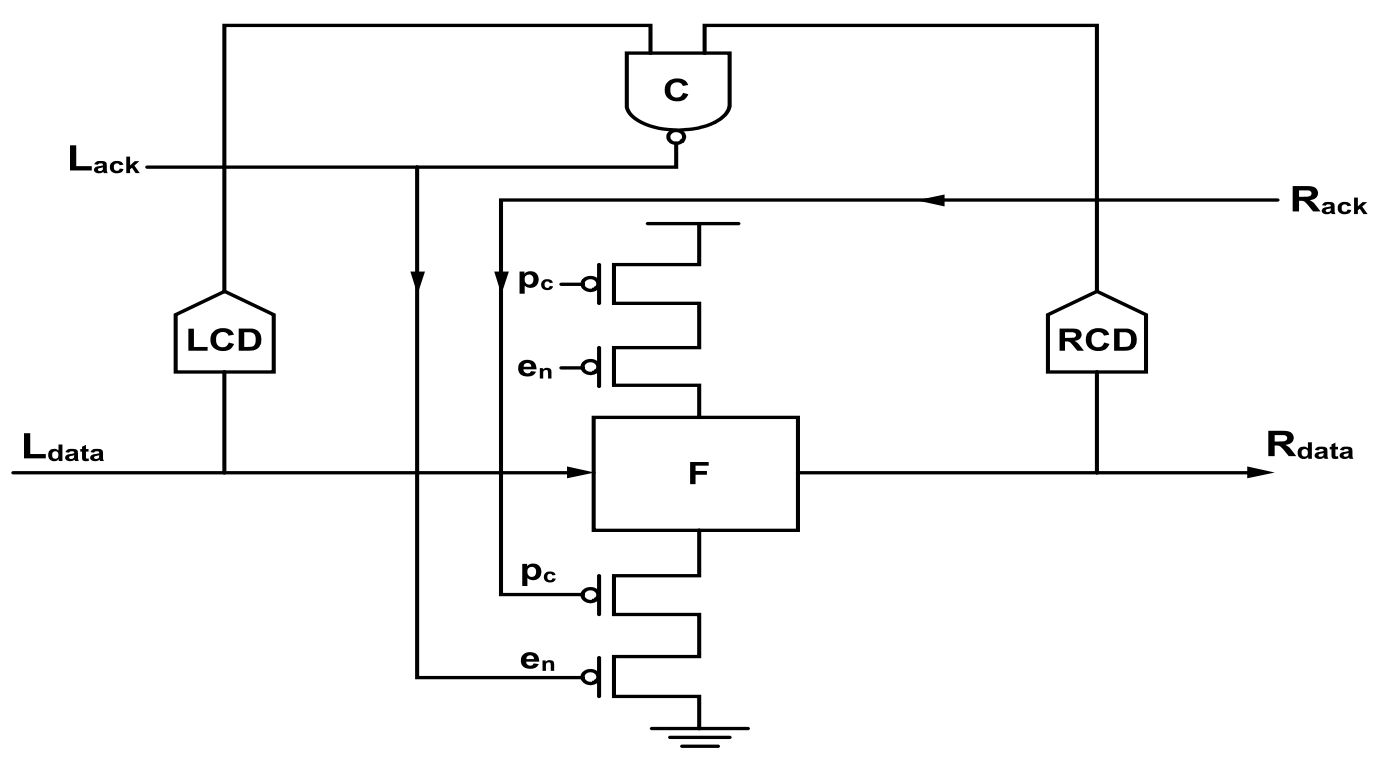

Fig 2:PCHB

In order for the functions block to evaluate before all the inputs have arrived, it need not be weak conditioned. On the other hand, the template only generates an acknowledgement signal Lack after all the inputs have arrived and output has been evaluated. The LCD and RCD are therefore combining using a C-element to generate the acknowledgement signal. Pointing out some aspects of this template; first, as the $\mathrm{C}$-element is inverting

the acknowledgement Signal is an active low signal. Second the Lack signal is after buffering using the inverters before being sent out usually, two other inverters are added to buffer the internal signal en that controls the function block.A WCHB is handy for buffers, but the precharge half buffer ( $\mathrm{PCHB}$ ) is

preferred for buffered logic. The PCHB template for two inputs and one output is shown in Figure 2 the PCHB template has a latency of two transitions and a cycle time of 14 transitions. The main difference from the WCHB is that the neutrality of the inputs is detected on Lack $\downarrow$ rather than Rack $\downarrow$. As a result, input neutrality can be detected in multiple transitions without impacting latency, which allows for a greater number of inputs. The Fig. 3 below shows the template for Pre-Charged Full Buffer (PCFB).The PCFB is more concurrent than the PCHB based on its $\mathrm{L}$ and $\mathrm{R}$ handshakes reset in parallel at the cost of requiring an additional state variable. 


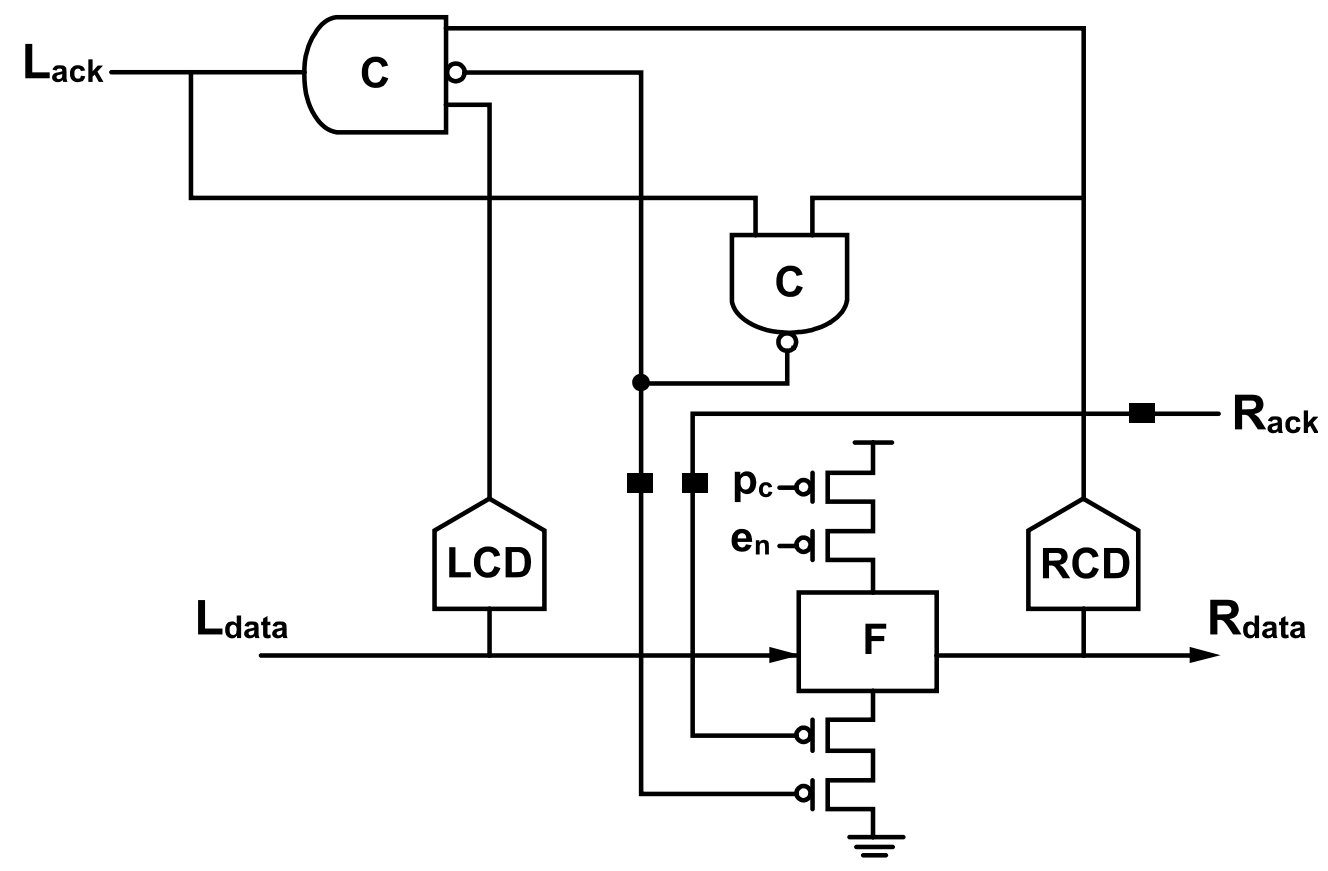

Fig 3: PCFB

Proposing a new pipeline template eliminating the need of the internal en signal of the PCHB template reducing the transition stack sizes in the function block. This new QDI pipeline template is referred to as a Reduced Stack PreCharged Half Buffer (RSPCHB) as shown in Fig.4.It is noted that the RCD block is optimized by tapping its inputs before the output inverter and a NAND gate is used instead of an OR gate. RSPCHB template facilitates the removal of the internal enable signal by reducing concurrency that does not improve performance. In the PCHB templates the output of LCD and RCD are companied using a C-element to generate acknowledgment signal Lack. As a result integration of the handshake protocol with the validity and neutrality of both input and output data is supported .This gets rid of the need for function block to be weak conditioned .Though requires the use of the en signal, this replacement introduces more concurrent than is necessary. In the case of a join, the non weak conditioned function block may generate an output when one of the input channels provides data. Therefore the RCD of the join asserts its output. On the other hand any subsequent stage can receive data, evaluate asserts both its LCD and RCD outputs and asserts acknowledgment signal. Though the join can receive acknowledgment, it will not precharge until after en is asserted. After the acknowledgment to the input stages has been asserted the en signal delays the precharge of the circuit. 


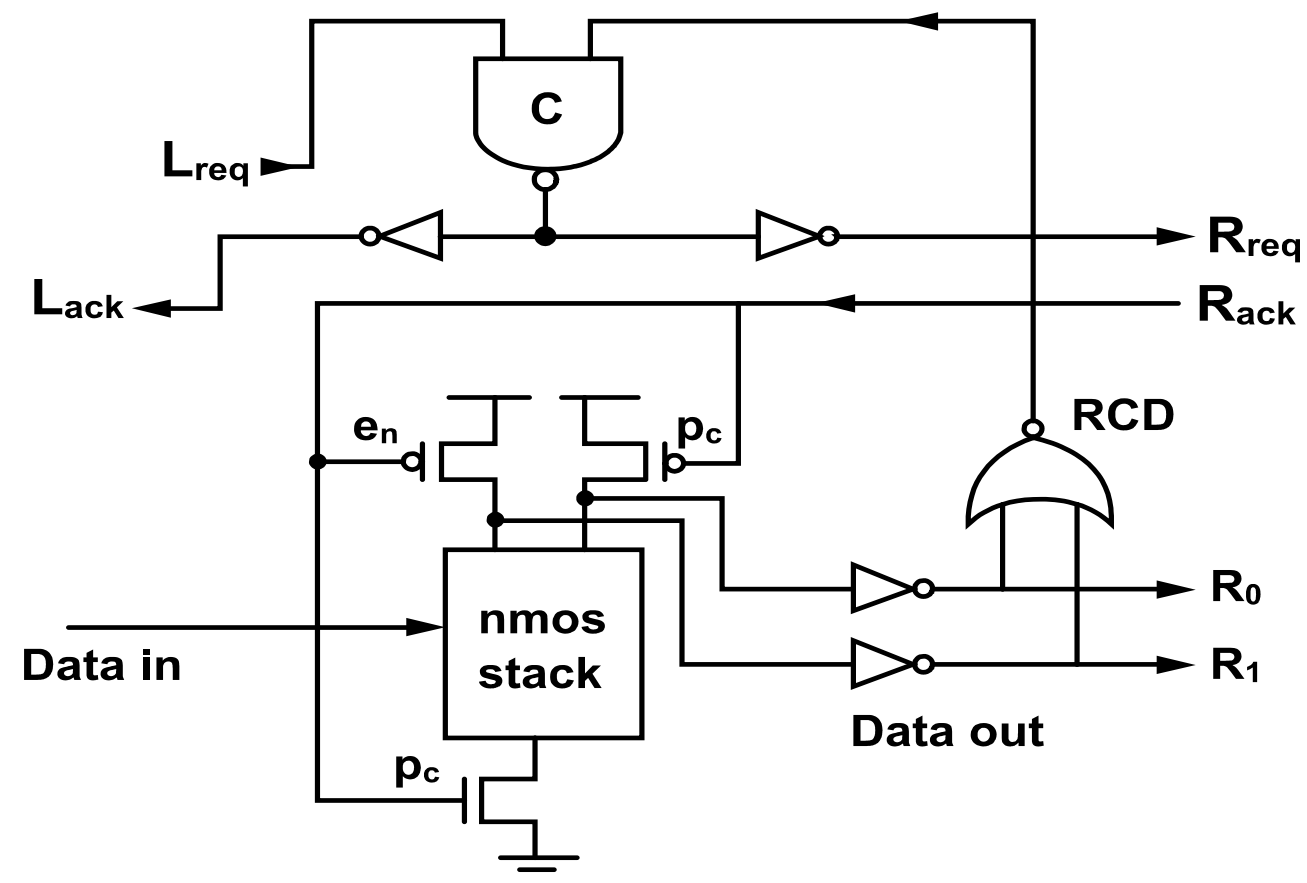

Fig 4: RSPCHB

This delay prevents the precharge from triggering the RCD to deassert. Further preventing the $\mathrm{C}$-element from ever generating the acknowledgment. The en signal could be safely removed if generation of acknowledgment signals from any stage subsequent to the join has arrived and been acknowledged .The join been the performance bottleneck for the subsequent stages, delay of the acknowledgment would not impact performance. The advantage of RSPCHB is that the lack of an LCD and reduced stack size of the function block, which reduces capacitive load and yields significantly faster overall performance. The cost of this increase in performance is that it requires one extra communicating wire between stages.

\section{Result}

The latency of the five standard circuits for the PCFB, PCHB and RSPCHB templates are shown in Figure 5.

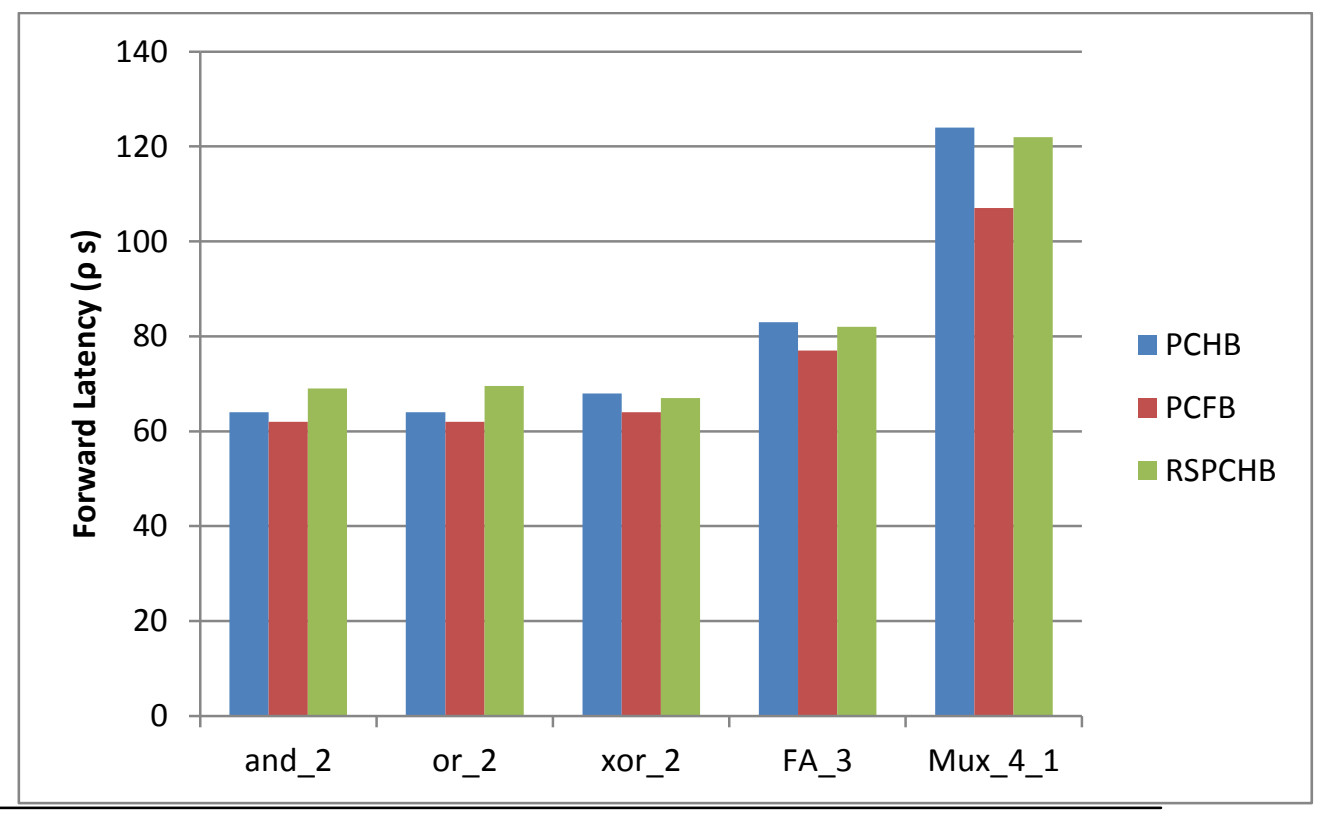

Fig 5.Forward latency of standard circuits across PCFB, PCHB, and RSPCHB templates 
On average, the latency of the PCHB is $6.5 \%$ less than the other circuit templates. The PCHB generally offer lower latency based on Re and Le are combined in a separate celement, rather than in the data rail stacks. The RSPCHB has a similar latency to the PCFB except and_2 and or_2 circuits where it's $6.5 \%$ slower.

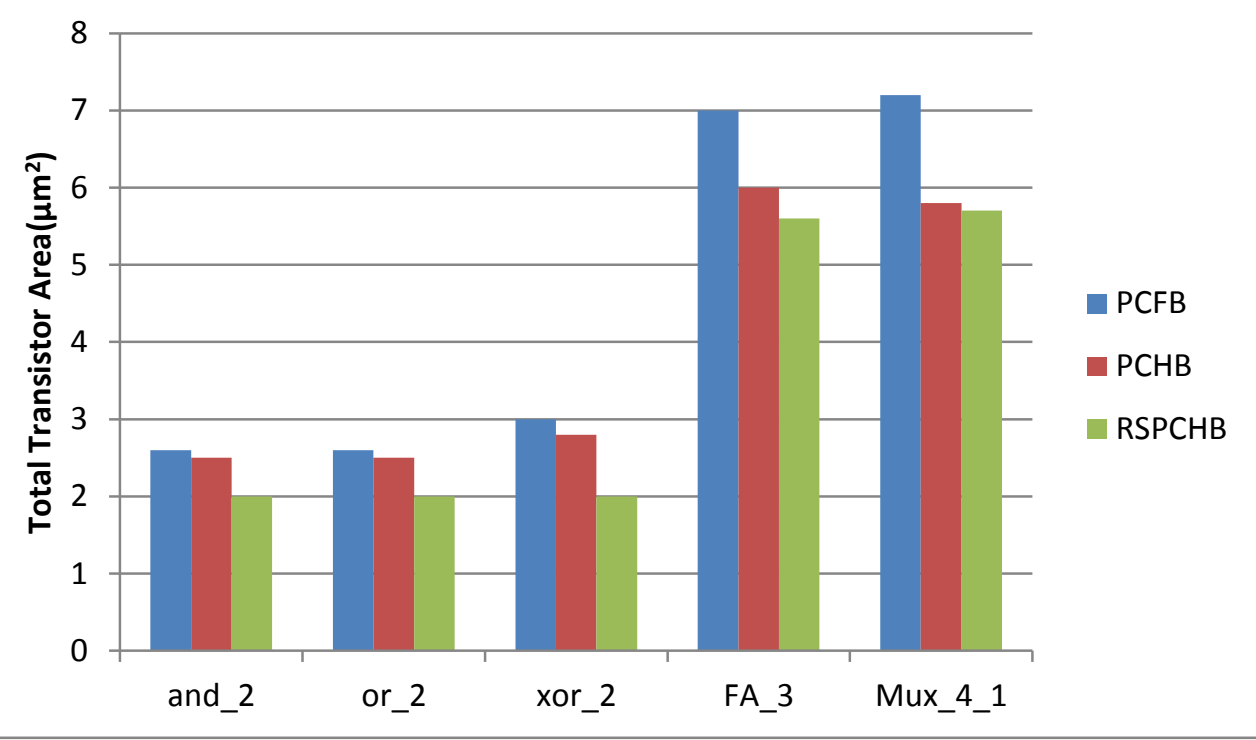

Fig 6.Total transistor area of standard circuits across PCFB, PCHB and RSPCHB templates

The pull down stacks in these circuits was augmented to wait for input validity, which makes them slower. Figure 6 shows a comparison of the total transistor area across the standard circuits. An interesting result is that the PCHB is slightly smaller than the PCFB. Once again, this is attributed to its simpler data rail transistor stacks. The RSPCHB is about $15.7 \%$ smaller than the PCHB template and $20.3 \%$ smaller than the PCFB template on average. This is a result of the simplified detection of input neutrality possible with the half cycle timing assumption.

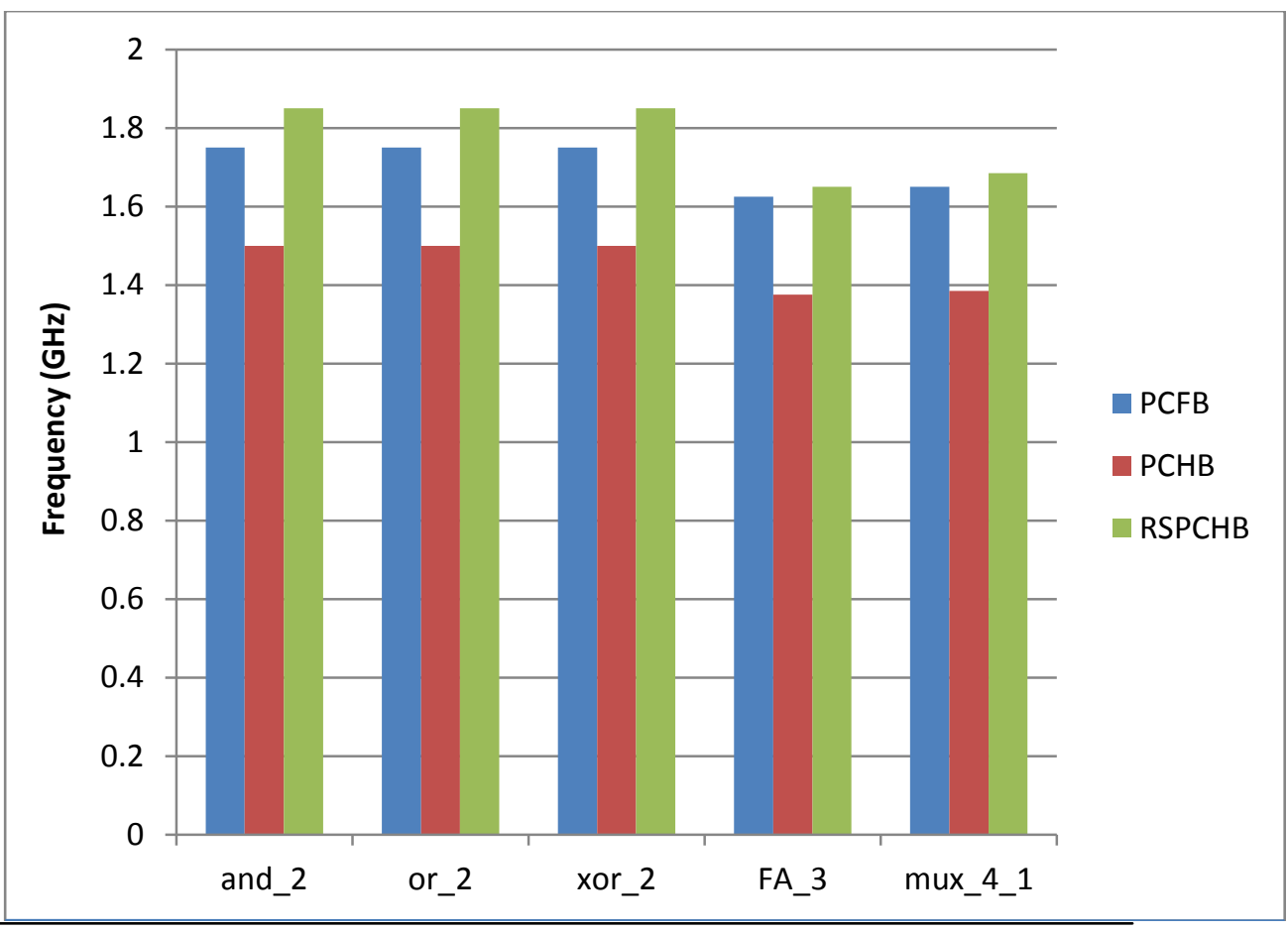

Fig 7. Frequency of standard circuits across PCFB, PCHB, and RSPCHB templates. 
The HCFB template is consistently higher frequency than the other templates across all five standard circuits, as seen in Figure 7. On average, the HCFB is $7.5 \%$ higher frequency than the PCFB. The PCHB has an 18 transition cycle time and the RSPCHB and PCFB both have a 14 transition cycle time. However, the RSPCHB offer higher frequency based on many of its transitions, especially those that detect input neutrality, are simpler. This suggests that HCFB can use even less area and smaller transistors for these fast transitions to match the frequency

\section{PERFORMANCE EVALUVATION}

Simulations are done with HSpice e using model files with $64.5 \mathrm{~nm}$ process. Each output node added by $4 \mathrm{fFwire}$ capacitance. This amount of capacitance is typical of short of the PCFB.The energy per operation (or per cycle) of the standard circuits is reported in Figure 8. The RSPCHB template consistently uses less energy than the PCFB and PCHB templates across all five standards. The RSPCHB template consumes $30 \%$ and $34 \%$ less energy on average than the PCFB and PCHB templates respectively. This is comparatively good based on its significant area savings, a slight frequency improvement, and a negligible latency penalty

wires based on extracted layout in this technology. Gates are sized to have the drive strength of an inverter with its pmos width set to 20 lambda units and its nmos width set to 10 lambda units(lambda is defined as half the minimum gate length). All power and energy numbers are based on total

power.

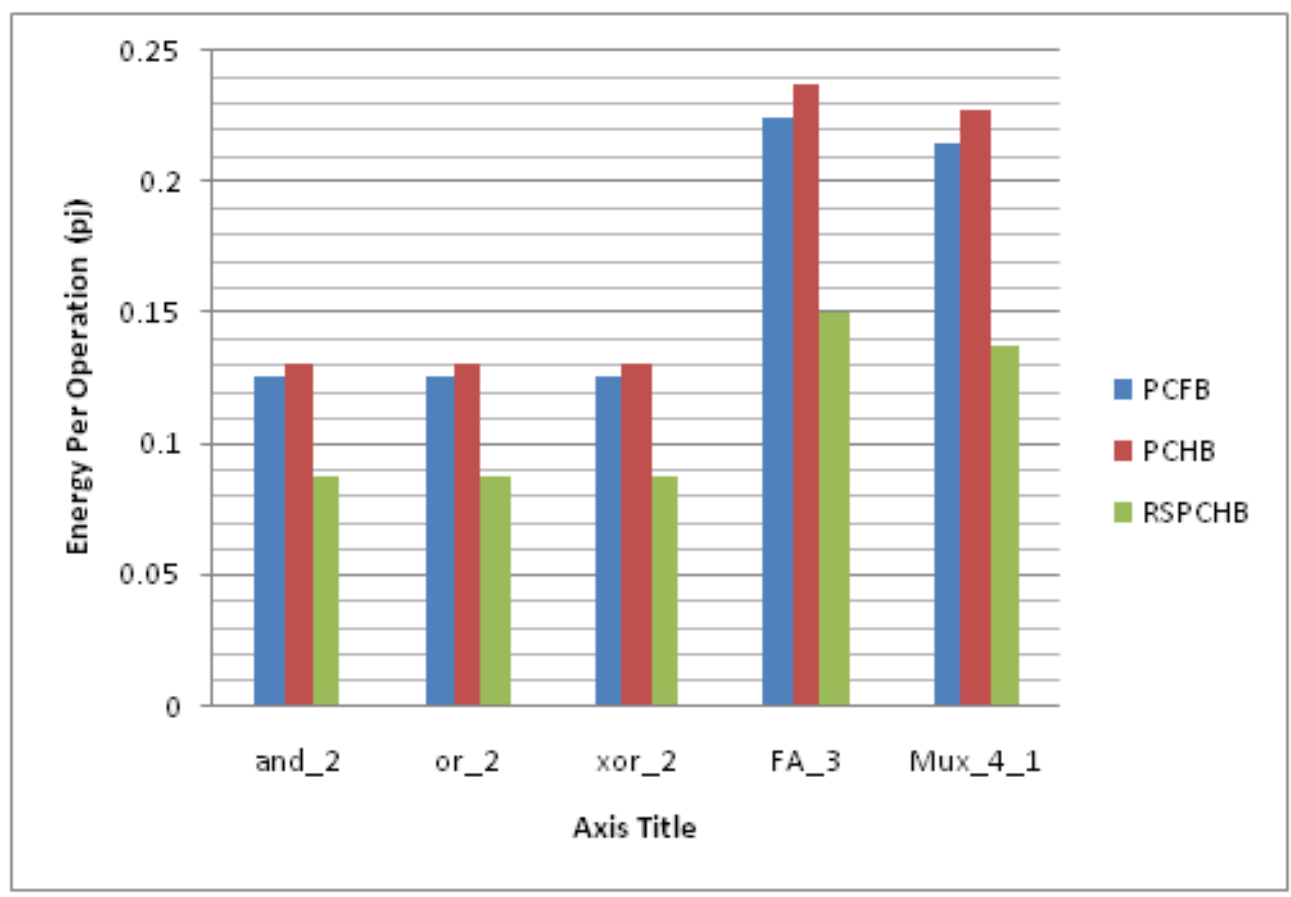

Fig. 8: Energy per operation of standard circuits across PCFB, PCHB, and RSCHB templates.

\section{CONCLUSION}

We investigated basic primitives with MSI circuits based on various asynchronous QDI predesigned templates. Using these styles, logic gates and some MSI circuits have been designed and simulated by Tanner EDA tool.

The author believe that the proposed design will provide a platform for designing high speed, low power digital circuits such as pipelined multiplier implemented in any application of digital signal processors.

\section{REFERENCES}

[1].Montek singh and steven M.Nowick The design of High performance dynamic asynchoronous pipelines; Lookahead style IEEE Transactions on Very Large Scale Integration systems.2007.

[2] Alain J. Martin ,Asynchronous Techniques for Systemon-Chip Design, Vol. 0018- 9219_2006.IEEE 94, No. 6 , June 2006 , Proceedings of the IEEE.

[3].D.M.Chapiro, Globally-Asynchronous,LocallySynchronous Systems, Ph.D. dissertation, Stanford 
Univ., Stanford, CA, 1984, Stanford CS Tech. Rep. STAN-CS-84-1026.

[4].LARS S. NIELSEN AND JENS SPARSO, Designing Asynchronous Circuits for Low Power:An IFIR Filter Bank for a Digital Hearing Aid, proceedings of the ieee, vol. 87, no. 2, February 1999.

[5].J. Cortadella et al., Logic Synthesis of Asynchronous Controllers and Interfaces. New York:SpringerVerlag, 2002.

[6]I.E.Sutherland,"Micropipelines," Commun. ACM, vol. 32, pp. 720-738,Jun 1989.

[7] Montek Singh and Steven M. Nowick, "MOUSETRAP: High-Speed Transition- Signaling Asynchronous Pipelines", IEEE transactions very on large scale integration (VLSI) systems, vol. 15, no. 6, June 2007.

[8]. Furber, S.B. and P. Day, 1996. Four-Phase Micropipeline Latch Control Circuits. IEEE Trans.
Very Large Scale Integration (VLSI) Syst., 4: 247253. DOI: $10.1109 / 92.502196$.

[9].Balasubramania, P., 2011. A Robust Asynchronous Early Output Full Adder. WSEAS, Trans. Circuits Syst., 10: 221-230.

[10]. LaFrieda, C. and R. Manohar, 2009. Reducing power consumption with relaxed quasi delay-insensitive circuits. Proceedings of the 15th IEEE Symposium on Asynchronous Circuits and Systems, May 17- 20, IEEE Xplore Press, Washington, USA, pp: 217-226. DOI: 10.1109/ASYNC.2009.9.

[11].AlainJ.Martin ,Asynchronous Techniques for Systemon-Chip Design, Vol. 0018- 9219_2006.IEEE 94, No. 6 , June 2006 , Proceedings of the IEEE.

[12] R. Manohar, "Width-adaptive data word architectures," in Adv. Res.VLSI, 2001, pp. 112-129. 